\title{
NMRI Mouse
}

National Cancer Institute

\section{Source}

National Cancer Institute. NMRI Mouse. NCI Thesaurus. Code C37416.

Derived from a Swiss type mouse that C. Lynch passed onto Poiley at NIH in 1937. This strain, then known as NIH/PI, was maintained as an inbred strain and was passed onto the Naval Medical Research Institute at F51. The NMRI mouse has a white coat color (albino) with genotype $\mathrm{A} / \mathrm{a}$, TyrC. 